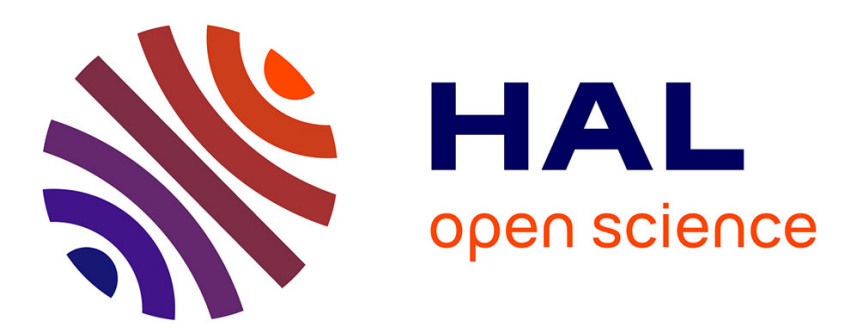

\title{
The Rheology of Carbon Nanotube (CNT) Suspensions: Experiments and Modelling
}

\author{
Anson W.K. Ma, Francisco Chinesta, Malcolm Mackley
}

\section{To cite this version:}

Anson W.K. Ma, Francisco Chinesta, Malcolm Mackley. The Rheology of Carbon Nanotube (CNT) Suspensions: Experiments and Modelling. XVth International Congress on Rheology, 2008, Monterey, United States. 10.1063/1.2964834 . hal-00290708

\section{HAL Id: hal-00290708 https://hal.science/hal-00290708}

Submitted on 28 Oct 2021

HAL is a multi-disciplinary open access archive for the deposit and dissemination of scientific research documents, whether they are published or not. The documents may come from teaching and research institutions in France or abroad, or from public or private research centers.
L'archive ouverte pluridisciplinaire HAL, est destinée au dépôt et à la diffusion de documents scientifiques de niveau recherche, publiés ou non, émanant des établissements d'enseignement et de recherche français ou étrangers, des laboratoires publics ou privés. 


\title{
The Rheology of Carbon Nanotube (CNT) Suspensions: Experiments and Modelling
}

\author{
Anson W. K. Ma ${ }^{a}$, Francisco Chinesta ${ }^{b}$ and Malcolm R. Mackley ${ }^{a}$ \\ " Department of Chemical Engineering, University of Cambridge, Pembroke Street, \\ Cambridge, CB2 3RA, United Kingdom \\ ${ }^{b}$ Laboratoire de Mécanique des Systèmes et des Procédés, \\ 151 Boulevard de l'Hôpital, 75013, Paris, France
}

\begin{abstract}
This paper reports the latest experimental results and rheological modelling for semi-dilute Carbon Nanotube (CNT) suspensions. CNTs belong to a relatively new class of nano-scale fibres having fascinating physical properties. In terms of experimental rheology, there are some similarities between CNT suspensions and classical carbon, glass, or polymeric fibre suspensions. Suspensions of CNTs with a Newtonian matrix were found to be shear-thinning in simple shear. The extent of shear-thinning, however, varies significantly depending on the type of CNTs used. As-produced untreated CNTs tended to give a much higher low-shear viscosity coupled with optically resolvable CNT aggregates, whereas CNTs that have been chemically treated showed little optical microstructure with less pronounced shear-thinning. Treated CNTs have been successfully modelled as short and rigid fibres that can align in the flow, but there are also randomising events due to Brownian rotary diffusion and tube-tube interactions. In the case of untreated CNTs, CNT orientation consideration alone was inadequate in explaining the experimentally observed shear thinning and a new model taking into account both elements of CNT orientation and aggregation kinetics has been formulated to describe the experimental data.
\end{abstract}

Keywords: carbon nanotube, suspensions, rheology, experiment, modelling.

PACS: 61.48.De; 83.80.Hj.

\section{INTRODUCTION}

This paper highlights some of our recent findings in relation to the steady shear rheology of Carbon Nanotubes (CNTs) suspended in a Newtonian matrix, whilst further experimental and modelling details will be reported in future papers [1-2]. Carbon Nanotubes (CNTs) are nano-scale fibres that can potentially be used for high performance nano-composites and processing CNTs for such application involves dispersing them in a suspending medium. Over the past few years, the rheology of CNT suspensions has been extensively studied for both chemically treated and untreated CNT suspensions (see for example [3-6]). It has been found that both types of CNT suspensions exhibited steady shear-thinning characteristic but to a different extent. Figure 1 shows the flow curves and matching optical microstructures for epoxy, treated and untreated CNT suspensions. It is clear from these results that chemically treated suspensions with little optical microstructure showed less pronounced shear-thinning effect compared with untreated CNT suspensions where clear network of CNT (aggregate) was observed.

\section{TREATED CNT SUSPENSIONS - ORIENTATION MODEL}

In terms of modelling, the rheological responses of treated CNTs can be explained by considering treated CNTs as rigid, short fibres that can align in flow, but also subject to Brownian rotary diffusion. Based on earlier work by Batchelor and co-workers (see for example [7]), the constitutive equation for a dilute suspension with high-aspectratio rigid fibres can be written as:

$$
\boldsymbol{\sigma}=-P \mathbf{I}+2 \eta \mathbf{D}+\boldsymbol{\tau}^{f}
$$


where $P$ denotes the pressure, $\mathbf{I}$ is the unit tensor, $\eta$ is the suspending medium viscosity, $\mathbf{D}$ is the strain rate tensor. $\boldsymbol{\tau}^{f}$ is the anisotropic viscous component of the stress tensor due to the presence of fibres, , which is further given as:

$$
\left\{\begin{array}{c}
\boldsymbol{\tau}^{f}=2 \eta N_{p}(\mathbf{A}: \mathbf{D})+\beta D_{r}\left(\mathbf{a}-\frac{\mathbf{I}}{3}\right) \\
\mathbf{A}=\int_{S(0,1)}(\boldsymbol{\rho} \otimes \boldsymbol{\rho} \otimes \boldsymbol{\rho} \otimes \boldsymbol{\rho}) \psi(\boldsymbol{\rho}) d \boldsymbol{\rho} \\
\mathbf{a}=\int_{S(0,1)}(\boldsymbol{\rho} \otimes \boldsymbol{\rho}) \psi(\boldsymbol{\rho}) d \boldsymbol{\rho}
\end{array}\right.
$$

where $N_{p}$ is a scalar parameter that depends on the fibre concentration as well as the aspect ratio of fibres in dilute suspensions, $\mathbf{A}$ and $\mathbf{a}$ are the fourth-order and second order orientation tensors respectively, $\boldsymbol{\rho}$ is the unit vector aligned in the fibre axis direction, $S(0,1)$ represents the surface of the unit sphere where the fiber orientation is defined, $\psi(\boldsymbol{\rho})$ is the fiber orientation distribution and $\beta$ is a parameter that depends on the number concentration of CNTs and temperature. $D_{r}$ is the rotary diffusion coefficient and physically it represents randomising events such as Brownian motion and tube-tube hydrodynamic interactions.

In terms of the mathematical treatment of CNT orientation distribution, closure approximations were avoided and the FokkerPlanck equation [Eq. (3)] was used to ensure numerical accuracy (see for example [19]):

$$
\frac{d \psi}{d t}+\frac{\partial}{\partial \boldsymbol{\rho}}\left(\frac{d \boldsymbol{\rho}}{d t} \psi\right)=\frac{\partial}{\partial \boldsymbol{\rho}}\left(D_{r} \frac{\partial \psi}{\partial \boldsymbol{\rho}}\right)
$$

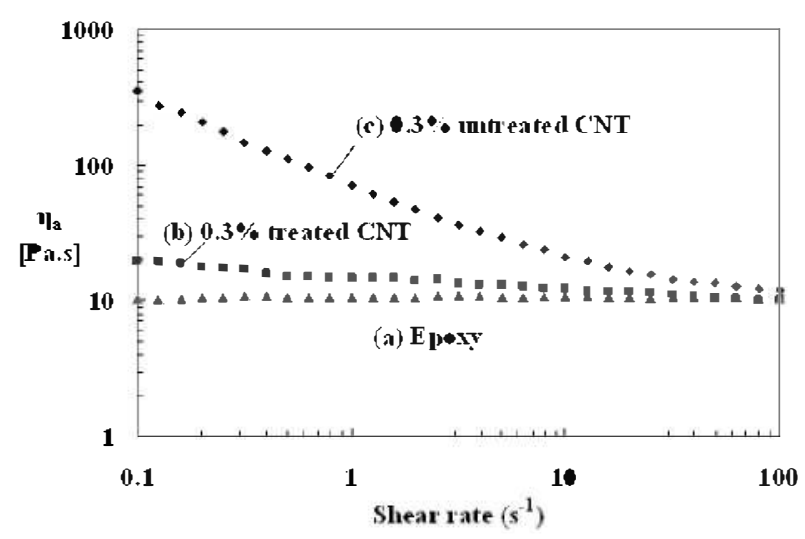

Eqs. (1-3) have been applied to the steady shear data

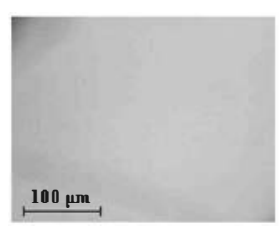

(a) $\mathrm{E}_{\mathrm{p}} \bullet \mathrm{xy}$

$\left(\right.$ Shear rate $\left.=\mathrm{s}^{-1}\right)$

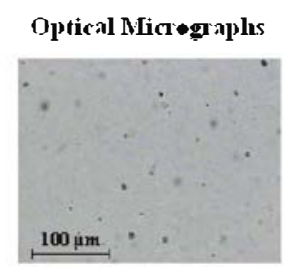

(b) $1.3 \%$ treated C'N T

(Shear rate $=\mathrm{s}^{\mathbf{1}}$ )

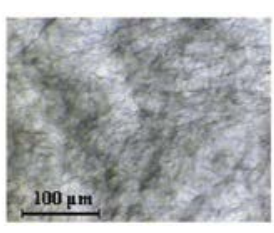

(c) $.3 \%$ untreaterl C'NT

(Shear rate $=\mathrm{s}^{-1}$ )

FIGURE 1 - Apparent steady shear viscosity $\left(\eta_{a}\right)$ and matching optical micrographs for (a) epoxy, (b) $0.3 \%$ treated and (c) $0.3 \%$ untreated CNT suspended in an epoxy resin (Araldite LY556, Huntsman LLC)

of treated CNT suspensions and Fig. 2 shows the model fitting of treated CNT suspensions with three different CNT concentrations $(0.05 \%, 0.2 \%$ and $0.33 \%)$. The rotary diffusion coefficient was identified to be in the order of $0.005 \mathrm{~s}^{-1}$ based on the best fits to experimental data. It has been found that the use of a constant $D_{r}$ appeared to be sufficient in providing a reasonably good estimation of the steady shear viscosity for general engineering problems.

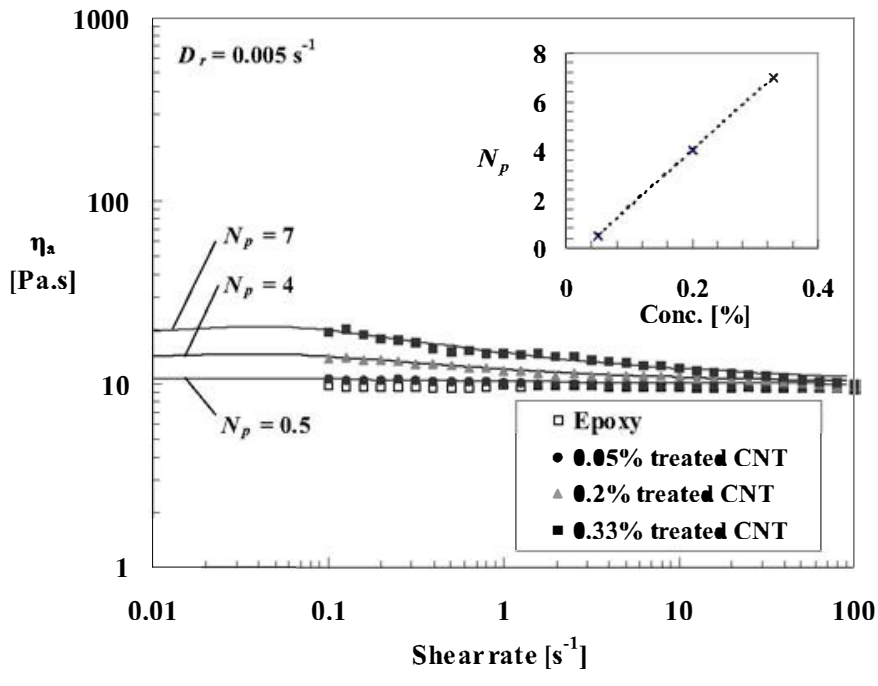

FIGURE 2 - Fokker-Planck based orientation model fitting of $0.05 \%, 0.2 \%$ and $0.33 \%$ treated CNT suspended in epoxy resin. The model consists of two fittings parameters: $D_{r}$ and $N_{p}$. Best fits were obtained using $D_{r}=0.005 \mathrm{~s}-1$ and the concentration-dependent parameter $N_{p}$ scaled linearly with the CNT concentration (inset figure). 


\section{UNTREATED CNT SUSPENSIONS - AGGREGATION/ORIENATION MODEL}

In the case of untreated suspensions, CNT orientation alone was found to be inadequate in explaining the experimentally observed rheological responses. A new model named the Aggregation/Orientation (AO) model was formulated to describe the experimental data. The model essentially considered a hierarchy of CNT aggregate structures in the untreated CNT suspension, where the shear viscosity was controlled not only by CNT orientation, but also the aggregation state of CNTs in the suspension. The Fokker-Planck description was modified to incorporate aggregation/disaggregation kinetics. Details on modifying the Fokker-Planck equation can be found in [2]. The resulting AO model contains three independent fitting parameters: the concentration and aspect-ratio parameter $\left(N_{p}^{\max }\right)$, the rotational diffusion coefficient $\left(D_{r}^{\max }\right)$ and the ratio between the aggregation and disaggregation velocities $\left(\beta=v_{c} / v_{\alpha}\right)$. Figure 3 shows the best fits to experimental data with two different CNT concentrations. In these fittings, it was assumed that only $N_{p}^{\max }$ depended on the CNT concentration and the other two rheological parameters were not a function of the concentration. Reasonable agreement between experimental results and the AO model was obtained with $D_{r}^{\max }=0.001 \mathrm{~s}^{-1}$ and $\beta=0.004$, further supporting the belief that the more pronounced shear-thinning in untreated CNT suspensions was due to the orientation as well as the aggregation of CNTs.
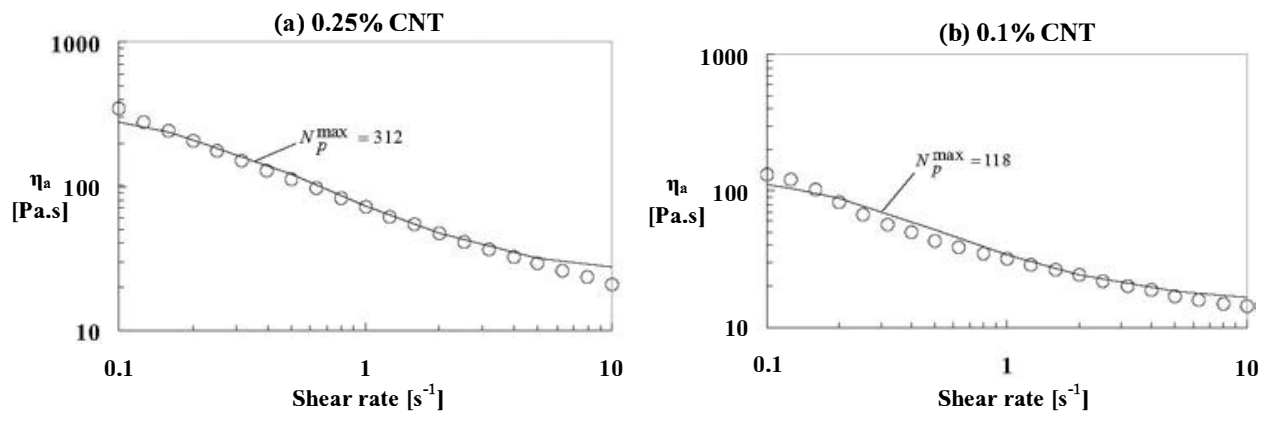

FIGURE 3 -

Aggregation/Orientation (AO) model fitting to experimental data of untreated CNT suspensions at two different CNT concentrations: (a) $0.25 \%$ and (b) $0.1 \%$. $D_{r}^{\max }=0.001 s^{-1}$ and $\beta=0.004 \cdot[2]$

\section{CONCLUSIONS}

The steady shear-thinning characteristics of both chemically treated and untreated Carbon Nanotube (CNT) suspensions have been modelled. Experimentally, untreated CNT suspensions were found to exhibit a more pronounced shear-thinning effect compared with treated CNT suspensions. The comparatively moderate shearthinning behaviour of treated CNT suspensions was successfully modelled using a Fokker-Planck based orientation model with two fitting parameters $\left(N_{p}\right.$ and $\left.D_{r}\right)$. The model essentially assumed treated CNTs as short and rigid fibres that can align in shear flow and shear thinning was believed to be a result of progressive alignment of CNTs in the flow direction. In the case of untreated CNT suspensions, the Fokker-Planck orientation model failed to describe the experimental shear thinning. A new model named Aggregation/Orientation (AO) model considering the effect of both CNT orientation and aggregation has been formulated. The resulting AO model with three independent fitting parameters was capable of describing experimental data of untreated CNT suspensions, offering a plausible explanation to the significant shear-thinning characteristic of aggregating untreated CNT suspensions.

\section{REFERENCES}

1. A. W. K. Ma, F. Chinesta, M. R. Mackley, J. Rheøl., in review.

2. A. W. K. Ma, F. Chinesta, A. Ammar, M. R. Mackley, J. Rheel., in review.

3. S. S. Rahatekar, et al., J. Rheøl. 50, $599-610$ (2006).

4. A. W. K. Ma, M. R. Mackley, S. S. Rahatekar, Rheøl. Act 46, 979 - 987 (2007).

5. A. W. K. Ma, F. Chinesta, T. Tuladhar, M. R. Mackley, Rheøl. Acta, published online (2008).

6. J. Xu, S. Chatterjee, K. W. Koelling, Y. Wang, S. E. Bechtel, Rheol. Act 44, $537-562$ (2005).

7. G. K. Batchelor, J. Fluid Mech. 41, $545-570$ (1970). 